\title{
Einstein-Podolsky-Rosen-Bohm laboratory experiments: Data analysis and simulation ${ }^{1}$
}

\author{
H. De Raedt*, K. Michielsen ${ }^{\dagger}$ and F. Jin ${ }^{\dagger}$ \\ *Department of Applied Physics, Zernike Institute for Advanced Materials, \\ University of Groningen, Nijenborgh 4, NL-9747 AG Groningen, The Netherlands \\ ${ }^{\dagger}$ Institute for Advanced Simulation, Jülich Supercomputing Centre, \\ Research Centre Jülich, D-52425 Jülich, Germany
}

\begin{abstract}
Data produced by laboratory Einstein-Podolsky-Rosen-Bohm (EPRB) experiments is tested against the hypothesis that the statistics of this data is given by quantum theory of this thought experiment. Statistical evidence is presented that the experimental data, while violating Bell inequalities, does not support this hypothesis. It is shown that an event-based simulation model, providing a cause-and-effect description of real EPRB experiments at a level of detail which is not covered by quantum theory, reproduces the results of quantum theory of this thought experiment, indicating that there is no fundamental obstacle for a real EPRB experiment to produce data that can be described by quantum theory.
\end{abstract}

Keywords: Einstein-Podolsky-Rosen-Bohm experiment, quantum theory, discrete event simulation

PACS: 03.65.Ud, 03.65.-w, 02.70.-c

\section{INTRODUCTION}

In the scientific and popular literature, it is common to find statements that EinsteinPodolsky-Rosen-Bohm (EPRB) experiments show violations of a Bell inequality and that the experimental results [1-13] are in favour of quantum theory [3, 14, 15]. While there can be little doubt that the former is a firmly established fact, it is remarkable that, at least to our knowledge, there seems to be no record of an hypothesis test that the experimental data gathered in EPRB experiments is indeed complying with the predictions of quantum theory for this particular experiment.

One reason for deeming such a test unnecessary may be rooted in the widespread believe that Bell $[14,16]$ would have proven that a violation of one of his inequalities implies that the experimental findings rule out any explanation in terms of classical (Hamiltonian as well as non-Hamiltonian) models that satisfy Einstein's criteria for local causality. Although the general validity of Bell's result has been questioned by many workers [17-48], even if the result were valid, it still remains to be shown that the experimental data is in concert with the predictions of quantum theory.

In this paper, we report on such an hypothesis test applied to data produced by the EPRB experiment of Weihs et al. [7, 8]. We present compelling evidence that the

\footnotetext{
${ }^{1}$ FPP6 - Foundations of Probability and Physics 6, edited by A. Khrennikov et al., (AIP Conference Proceedings, Melville and New York, in press)
} 


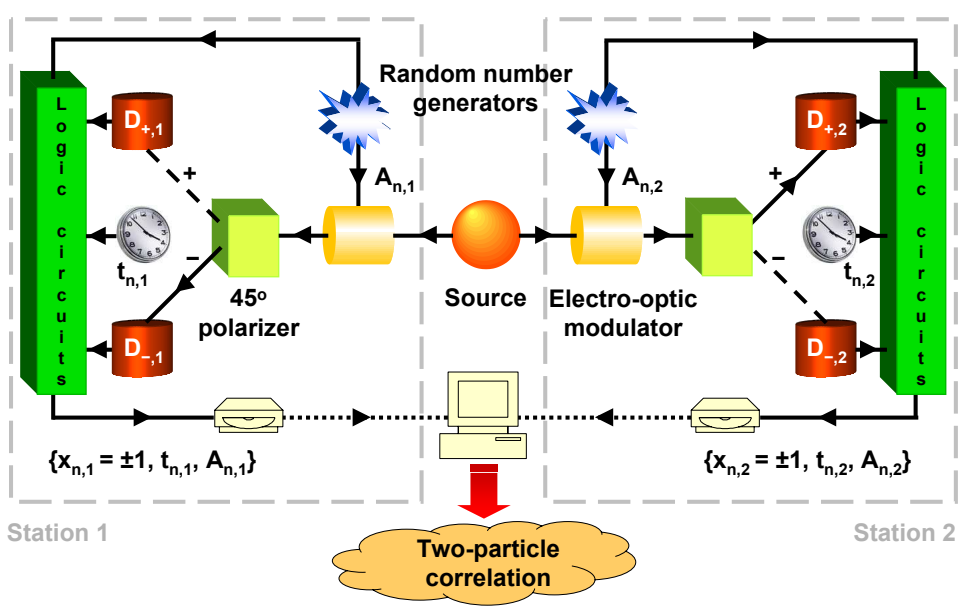

FIGURE 1. Schematic diagram of an EPRB experiment with photons [7, 8].

experimental data, while violating Bell inequalities, does not support the hypothesis that the data complies with the predictions of quantum theory for the EPRB experiment. We also demonstrate that classical, locally causal models which generate the same kind of data sets as the laboratory EPRB experiments can reproduce the results of quantum theory, suggesting that in the real experiments there are processes at work which deserve to be identified and studied further.

The data sets generated by the experiment of Weihs et al. have been scrutinized earlier [13, 40, 49-51]. A. Hnilo et al. focused on ruling out certain types of local realist models and searched for non-ergodic features in the time-stamped data $[13,49,51]$. G. Adenier and A. Khrennikov tested the hypothesis that the data of the experiment of Weihs et al. supports the fair sampling assumption [40]. They showed that the relative frequencies of single-particle counts exhibits "non-local" dependencies on the applied bias and interpret this finding as evidence that the fair sampling assumption may be violated in the experiments of Weihs et al. [40]. Their conclusions were criticized by J.H. Bigelow [50] who showed that, with additional assumptions, the violation of the non-signaling criterion, found by G. Adenier and A. Khrennikov, can be removed by modelling the data as a fair sample drawn from a set of sixteen coincidence counts that obey the no-signaling criterion.

\section{EPRB LABORATORY EXPERIMENT}

In Fig. 1, we show a schematic diagram of the EPRB experiment with photons [7, 8], the data of which we analyze. In this experiment, the polarization of each photon is used as the spin-1/2 degree-of-freedom in Bohm's version [52] of the EPR gedanken experiment [53].

The source is emitting pairs of photons. The statistical properties of a large set of these photons are a priori unknown and have to be inferred from the analysis of the recorded detection events. As the photon arrives at station $i=1,2(i=1,2$ denotes Alice's and 
Bob's station, respectively), it passes through an electro-optic modulator (EOM). The EOM in observation station $i$ rotates the polarization of the photon that passes through it by an amount that is controlled by a voltage applied to the EOM [7, 8]. In turn, this voltage is controlled by a binary variable $A_{i}$, which is chosen at random $[7,8]$. Optionally, a bias voltage is added to the randomly varying voltage $[7,8]$. The relation between the voltage applied to the EOM and the resulting rotation of the polarization is determined experimentally, hence there is some uncertainty in relating the applied voltage to the rotation angle $[7,8]$. Keeping in mind that the experimentally relevant variable is the applied voltage, to simplify the presentation and the interpretation in terms of quantum theory, we characterize the rotation of the polarization by the angle $\theta_{i}$, as in Refs. [7, 8]. As the photon leaves the EOM, a polarizing beam splitter directs the photon to one of the two detectors, producing a signal $x_{n, i}= \pm 1$ where the subscript $n$ labels the $n$th detection event (see Fig. 1). Each station has its own clock that assigns a time-tag to each signal generated by one of the two detectors [7, 8]. Effectively, this procedure discretizes time in intervals, the width of which is determined by the time-tag resolution $\tau$. In the experiment, the time-tag generators are synchronized before each run $[7,8]$. The firing of a detector defines an event. At the $n$th event at station $i$, the dichotomic variable $A_{n, i}$ (see Fig. 1), controlling the rotation angle $\theta_{n, i}$, the dichotomic variable $x_{n, i}$ designating which detector fires, and the time tag $t_{n, i}$ of the detection event are written to a file on a hard disk, allowing the data to be analyzed long after the experiment has terminated $[7,8]$. The set of data collected at station $i$ may be written as

$$
\Upsilon_{i}=\left\{x_{n, i}, t_{n, i}, \theta_{n, i} \mid n=1, \ldots, N_{i}\right\}
$$

where we allow for the possibility that the number of detected events $N_{i}$ at stations $i=$ 1,2 need not (and in practice is not) to be the same and we have used the rotation angle $\theta_{n, i}$ instead of the corresponding dichotomic variable $A_{n, i}$ to facilitate the comparison with the quantum theoretical description. The data sets $\left\{\Upsilon_{1}, \Upsilon_{2}\right\}$, kindly provided to us by $\mathrm{G}$. Weihs, are the starting point for the analysis presented in this paper.

A laboratory EPRB experiment requires some criterion to decide which detection events are to be considered as stemming from a single or two-particle system. In EPRB experiments with photons, this decision is taken on the basis of coincidence in time [7, 54]. Here we adopt the procedure employed by Weihs et al. [7, 8]. Coincidences are identified by comparing the time differences $t_{n, 1}-t_{m, 2}$ with a window $W[7,8,54]$, where $n=1, \ldots, N_{1}$ and $m=1, \ldots, N_{2}$. By definition, for each pair of rotation angles $a$ and $b$, the number of coincidences between detectors $D_{x, 1}(x= \pm 1)$ at station 1 and detectors $D_{y, 2}(y= \pm 1)$ at station 2 is given by

$$
C_{x y}=C_{x y}(a, b)=\sum_{n=1}^{N_{1}} \sum_{m=1}^{N_{2}} \delta_{x, x_{n, 1}} \delta_{y, x_{m, 2}} \delta_{a, \theta_{n, 1}} \delta_{b, \theta_{m, 2}} \Theta\left(W-\left|t_{n, 1}-t_{m, 2}\right|\right),
$$

where $\Theta(t)$ is the Heaviside step function. In Eq. (2) the sum over all events has to be carried out such that each event (= one detected photon) contributes only once. Clearly, this constraint introduces some ambiguity in the counting procedure as there is a priori, no clear-cut criterion to decide which events at stations $i=1$ and $i=2$ should be paired. One obvious criterion might be to choose the pairs such that $C_{x y}$ is maximum 
but as we explain later, such a criterion renders the data analysis procedure (not the data production!) acausal. It is trivial though (see later) to analyse the data generated by the experiment of Weihs et al. such that conclusions do not suffer from this artifact. In general, the values for the coincidences $C_{x y}(a, b)$ depend on the time-tag resolution $\tau$ and the window $W$ used to identify the coincidences.

The single-particle averages and correlation between the coincidence counts are defined by

$$
\begin{aligned}
E_{1}(a, b) & =\frac{\sum_{x, y= \pm 1} x C_{x y}}{\sum_{x, y= \pm 1} C_{x y}}=\frac{C_{++}-C_{--}+C_{+-}-C_{-+}}{C_{++}+C_{--}+C_{+-}+C_{-+}} \\
E_{2}(a, b) & =\frac{\sum_{x, y= \pm 1} y C_{x y}}{\sum_{x, y= \pm 1} C_{x y}}=\frac{C_{++}-C_{--}-C_{+-}+C_{-+}}{C_{++}+C_{--}+C_{+-}+C_{-+}} \\
E(a, b) & =\frac{\sum_{x, y= \pm 1} x y C_{x y}}{\sum_{x, y= \pm 1} C_{x y}}=\frac{C_{++}+C_{--}-C_{+-}-C_{-+}}{C_{++}+C_{--}+C_{+-}+C_{-+}}
\end{aligned}
$$

where the denominator $N_{c}=C_{++}+C_{--}+C_{+-}+C_{-+}$in Eq. (3) is the sum of all coincidences. In practice, coincidences are determined by a four-step procedure [8]:

1. Compute a histogram of time-tag differences $t_{n, 1}-t_{m, 2}$ of pairs of detection events.

2. Determine the time difference $\Delta_{G}$ for which this histogram shows a maximum.

3. Add $\Delta_{G}$ to the time-tag data of Alice, thereby moving the position of the maximum of the histogram to zero.

4. Determine the coincidences using the new time-tag differences, each photon contributing to the coincidence count at most once.

The global offset, denoted by $\Delta_{G}$, may be attributed to the loss of synchronization of the clocks used in the stations of Alice and Bob [8].

\section{Role of the time window and acausal data processing}

Most theoretical, local-realistic treatments of the EPRB experiment assume that the correlation, as measured in the experiment, is given by [14]

$$
C_{x y}^{(\infty)}(a, b)=\sum_{n=1}^{N} \delta_{x, x_{n, 1}} \delta_{y, x_{n, 2}} \delta_{a, \theta_{n, 1}} \delta_{b, \theta_{m, 2}}
$$

which is obtained from Eq. (2) by taking the limit $W \rightarrow \infty$ and omitting events such that $N=N_{1}=N_{2}$. A naive argument that might justify taking this limit is the hypothesis that for ideal experiments, the value of $W$ should not matter because the time window only serves to identify pairs. However, this argument does not apply to real experiments: The analysis of the data of the experiment of Weihs et al. shows that the average time between pairs of photons is of the order of $30 \mu$ s or more, much larger than the typical values (of the order of a few nanoseconds) of the time-window $W$ used in the experiments [8]. In other words, in practice, the identification of photon pairs does not require the use 
of $W$ 's of the order of a few nanoseconds in which case the use of a time-coincidence window does not create a "loophole". A narrow time window mainly acts as a filter that selects pairs, the photons of which experienced time delays that differ by the order of nanoseconds.

The use of a global offset $\Delta_{G}$, determined by maximizing the number of coincidences, introduces an element of non-causality in the analysis of the experimental results (but not in the original data itself): Whether or not at a certain time, a pair contributes to the number of coincidences depends on all the coincidences, also on those at later times. This is an example of an acausal filtering [55]: The output (coincidence or not) depends on both all previous and all later inputs (the detection events and corresponding time-tags). Therefore, data analysis of EPRB experiments that employs a global offset $\Delta_{G}$ to maximize the number of coincidences, is intrinsically acausal: The notion of coincidences happening inside or outside the light cone becomes irrelevant.

In spite of this caveat, very useful information can be extracted from data sets produced by the experiment of Weihs et al., the reason being that the results of the analysis become independent of $\Delta_{G}$ if the time-window $W$ is taken to be sufficiently large (but much smaller than the average time between successive events). As it is our aim to test whether the data of the experiment of Weihs et al. comply with quantum theory, not to find the maximum violation of some inequality, we will not dwell on this issue any further and simply discard conclusions that depend on the use of a non-zero $\Delta_{G}$.

\section{HYPOTHESIS TEST}

According to quantum theory of the EPRB thought experiment, the results of repeated measurements of the system of two $S=1 / 2$ particles in the spin state $|\Phi\rangle$ are given by the single-spin expectation values

$$
\begin{aligned}
& \widehat{E}_{1}(a)=\left\langle\Phi\left|\mathbf{S}_{1} \cdot \mathbf{a}\right| \Phi\right\rangle=\left\langle\Phi\left|\mathbf{S}_{1}\right| \Phi\right\rangle \cdot \mathbf{a} \\
& \widehat{E}_{2}(b)=\left\langle\Phi\left|\mathbf{S}_{2} \cdot \mathbf{b}\right| \Phi\right\rangle=\left\langle\Phi\left|\mathbf{S}_{2}\right| \Phi\right\rangle \cdot \mathbf{b}
\end{aligned}
$$

and the two-particle correlations $\widehat{E}(a, b)=\left\langle\Phi\left|\mathbf{S}_{1} \cdot \mathbf{a} \mathbf{S}_{2} \cdot \mathbf{b}\right| \Phi\right\rangle=\mathbf{a} \cdot\left\langle\Phi\left|\mathbf{S}_{1} \mathbf{S}_{2}\right| \Phi\right\rangle \cdot \mathbf{b}$ where $\mathbf{a}=(\cos a, \sin a)$ and $\mathbf{b}=(\cos b, \sin b)$ specify the directions of the analyzers (corresponding to the rotations of the polarization due to the EOM's). We have introduced the notation $\widehat{~ t o ~ d i s t i n g u i s h ~ t h e ~ q u a n t u m ~ t h e o r e t i c a l ~ p r e d i c t i o n ~ f r o m ~ t h e ~ r e s u l t s ~ o b t a i n e d ~ b y ~}$ analysis of the experimental data sets.

Quantum theory of the EPRB thought experiment assumes that $|\Phi\rangle$ does not depend on $a$ or $b$. Therefore, from Eq. (5) it follows immediately that $\widehat{E}_{1}(a)$ does not depend on $b$ and that $\widehat{E}_{2}(b)$ does not depend on $a$. Under the hypothesis that quantum theory describes the data collected in the laboratory EPRB experiment, we may expect that $E_{1}(a, b) \approx \widehat{E}_{1}(a)$ and $E_{2}(a, b) \approx \widehat{E}_{2}(b)$, exhibit the same independencies. This is the basis of our test.

In practice, we are dealing with real data and not with mathematical expressions such as Eq. (5) and therefore, we need a criterion to decide whether or not the data is in 

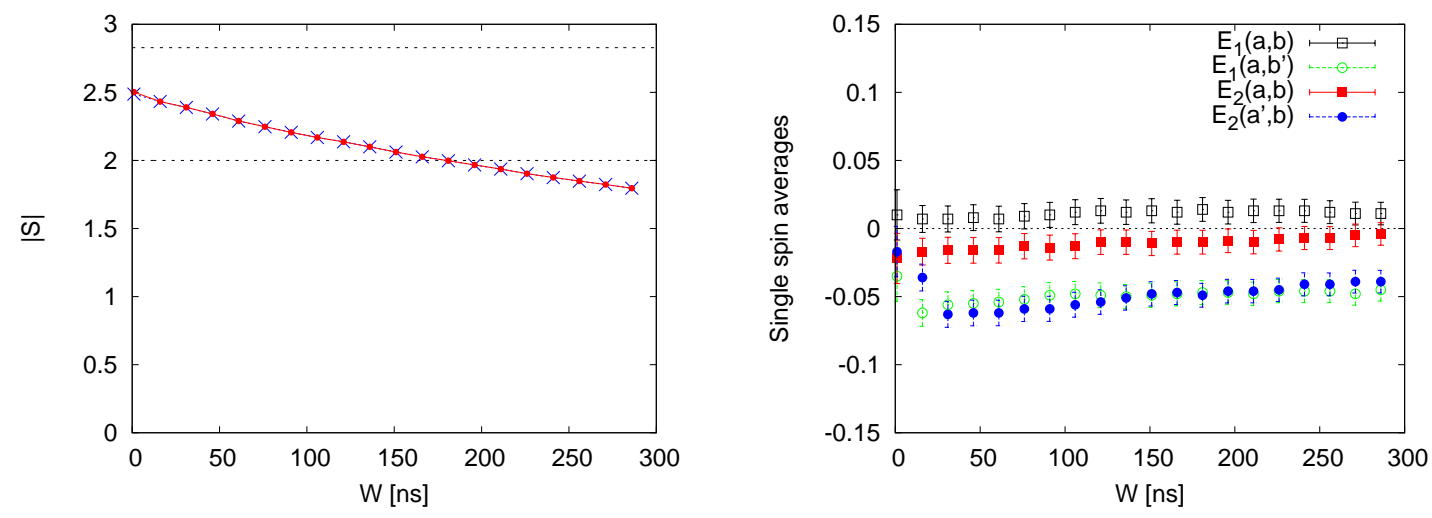

FIGURE 2. Analysis of the data set newlongtime2. Left: $|S|=\left|E(a, b)-E\left(a, b^{\prime}\right)+E\left(a^{\prime}, b\right)+E\left(a^{\prime}, b^{\prime}\right)\right|$ as a function of the time window $W$ and $a=0, a^{\prime}=\pi / 4, b=\pi / 8$ and $b^{\prime}=3 \pi / 8$. The dashed lines represent the maximum value for a quantum system of two $S=1 / 2$ particles in a separable (product) state $(|S|=2)$ and in a singlet state $(|S|=2 \sqrt{2})$, respectively. Blue crosses: $\Delta_{G}=0$. Red bullets connected by the red solid line: $\Delta_{G}=0.5 \mathrm{~ns}$. Right: Selected single-particle averages as a function of $W$ and for $\Delta_{G}=0$. The error bars correspond to 2.5 standard deviations. For small $W$, the total number of coincidences is too small to yield statistically meaningful results. For $W>20 \mathrm{~ns}$ the change of some of these single-spin averages observed by Bob (Alice) when Alice (Bob) changes her (his) setting, systematically exceeds five standard deviations, suggesting it is highly unlikely that the data is in concert with quantum theory of the EPRB experiment.

agreement with the value predicted by quantum theory of the EPRB experiment. In line with standard statistical hypothesis testing, we adopt the following criterion:

The data for $E_{1}(a, b)\left(E_{2}(a, b)\right)$ are considered to be in conflict with the prediction of quantum theory of the EPRB experiment if it shows a dependency on $b(a)$ that exceeds five times the upper bound $1 / \sqrt{N_{c}}$ to the standard deviation $\sigma_{N_{c}}$.

Data that does not satisfy our criterion exhibits a spurious kind of "non-locality" that cannot be explained by the quantum theoretical description of the EPRB experiment.

A key feature of our test is that it does not rely on any particular property of the state $|\Phi\rangle$. For instance, if in a laboratory EPRB experiment we find that $E_{1}(a, b)$ shows a dependence on $b$ that exceeds five times the standard deviation, this dependence cannot be attributed to $|\Phi\rangle$ deviating from the singlet state.

Figure 2(left) shows the typical results of the Bell function $S$ as a function of $W$. For $W<150 \mathrm{~ns}$, the Bell inequality $|S| \leq 2$ is clearly violated. For $W>200 \mathrm{~ns}$, much less than the average time ( $>30 \mu \mathrm{s}$ ) between two coincidences, the Bell inequality $|S| \leq 2$ is satisfied, demonstrating that the "nature" of the emitted pairs is not an intrinsic property of the pairs themselves but also depends on the choice of $W$ made by the experimenter. For $W>20 \mathrm{~ns}$, there is no significant statistical evidence that the "noise" on the data depends on $W$ but if the only goal is to maximize $|S|$, it is expedient to consider $W<20 \mathrm{~ns}$. From these experimental results, it is clear that the time-coincidence window does not constitute a "loophole". Not only is it an essential element of these EPRB experiments, it also acts as a filter that is essential for the data to violate a Bell inequality. 
TABLE 1. Results of the numerical solution of the set of three groups of equations such as Eq. (7) for data taken from the experimental data set newlongtime 2 at $W=50 \mathrm{~ns}$. The pair of settings that has not been included corresponds to the entry indicated with the -. For instance, the results of the first row have been obtained by excluding the data for the setting $\left(a^{\prime}, b^{\prime}\right)$. Consistency demands that the numbers in each column are close to each other. The fact that the four solutions are inconsistent suggests that the data is incompatible with quantum theory for the EPRB thought experiment.

\begin{tabular}{cccccccccc}
\hline$r_{1}$ & $r_{2}$ & $\widehat{E}_{1}(a)$ & $\widehat{E}_{1}\left(a^{\prime}\right)$ & $\widehat{E}_{2}(b)$ & $\widehat{E}_{2}\left(b^{\prime}\right)$ & $\widehat{E}(a, b)$ & $\widehat{E}\left(a, b^{\prime}\right)$ & $\widehat{E}\left(a^{\prime}, b\right)$ & $\widehat{E}\left(a^{\prime}, b^{\prime}\right)$ \\
\hline+0.17 & -0.01 & -0.17 & -0.23 & +0.11 & -0.13 & -0.72 & +0.47 & -0.52 & - \\
-0.06 & -0.01 & +0.06 & +0.03 & -0.05 & -0.03 & -0.69 & +0.45 & - & -0.71 \\
+0.17 & -0.16 & -0.29 & -0.32 & +0.27 & +0.31 & -0.83 & - & -0.62 & -0.87 \\
-0.06 & -0.17 & +0.13 & -0.08 & +0.16 & +0.13 & - & +0.46 & -0.50 & -0.72 \\
\hline
\end{tabular}

Figure 2(right) shows results of a selection of single-particle expectations as a function of $W$. It is clear that these results violate our criterion for being compatible with the prediction of quantum theory of the EPRB model. According to standard practice of hypothesis testing, the likelyhood that this data set can be described by the quantum theory of the EPRB experiment should be considered as extremely small. This finding is not an accident: Our analysis of 23 data sets produced by the experiment of Weihs et al. shows that none of these data sets satisfies our hypothesis test for being compatible with the predictions of quantum theory of the EPRB model.

\section{ROLE OF THE DETECTION EFFICIENCY}

For the experimental set up of Weihs et al., the dependence of $E_{1}(a, b)\left(E_{2}(a, b)\right)$ on $b$ (a) cannot be attributed to detection efficiencies of the detectors at station (1), assuming the latter as constant during the time the data is collected. This can be seen as follows.

As photons may be absorbed when passing through the EOM and as detectors do not register all incident photons, we may write

$$
C_{x y}(a, b)=\kappa_{1}(a) \kappa_{2}(b) \eta_{1}(x) \eta_{2}(y) N P(x y \mid a b)
$$

where $0<\kappa_{i}() \leq$.1 and $0<\eta_{i}() \leq$.1 represent the efficiency of the EOM and detectors at station $i=1,2$ respectively, $N$ is the number of photon pairs emitted by the source and $P(x y \mid a b)=\left(1+x \widehat{E}_{1}(a)+y \widehat{E}_{2}(b)+x y \widehat{E}(a, b)\right) / 4$ is the most general form of the probability for a pair $x, y= \pm 1$, compatible with quantum theory of the EPRB thought experiment. Note that the experiment of Weihs et al. uses polarizing beam splitters, and therefore the detectors receive photons with fixed polarization. Hence the detection efficiencies $\eta_{1}(x)$ and $\eta_{2}(y)$ are not expected to depend on the polarization of the photons that leave the EOMs [8]. After some elementary algebra, we find

$$
\begin{aligned}
& E_{1}(a, b)=\frac{r_{1}+\widehat{E}_{1}(a)+r_{1} r_{2} \widehat{E}_{2}(b)+r_{2} \widehat{E}(a, b)}{1+r_{2} \widehat{E}_{1}(a)+r_{1} \widehat{E}_{2}(b)+r_{1} r_{2} \widehat{E}(a, b)} . \\
& E_{2}(a, b)=\frac{r_{2}+r_{1} r_{2} \widehat{E}_{1}(a)+\widehat{E}_{2}(b)+r_{1} \widehat{E}(a, b)}{1+r_{2} \widehat{E}_{1}(a)+r_{1} \widehat{E}_{2}(b)+r_{1} r_{2} \widehat{E}(a, b)},
\end{aligned}
$$




$$
E(a, b)=\frac{r_{1} r_{2}+r_{2} \widehat{E}_{1}(a)+r_{1} \widehat{E}_{2}(b)+\widehat{E}(a, b)}{1+r_{2} \widehat{E}_{1}(a)+r_{1} \widehat{E}_{2}(b)+r_{1} r_{2} \widehat{E}(a, b)},
$$

where $-1 \leq r_{i}=\left(\eta_{i}(+1)-\eta_{i}(-1)\right) /\left(\eta_{i}(+1)+\eta_{i}(-1)\right) \leq 1$ parameterizes the relative efficiencies of the detectors at stations $i=1,2$. The parameters $r_{1}, r_{2}$ and the state of the quantum system (fully described by $\widehat{E}_{1}(a), \widehat{E}_{2}(b), \widehat{E}(a, b)$ ) can be determined by considering the set of nine equations for the three pairs of settings. Taking for instance the data for the set of settings $\left\{(a, b),\left(a, b^{\prime}\right),\left(a^{\prime}, b\right)\right\}$, yields three times three equations of the form Eq. (7) with nine unknowns which are easily solved numerically. As an illustrative example, we examine the data at $W=50 \mathrm{~ns}$ taken from the experimental data set newlongtime2, see also Fig. 2. The results of the numerical solution of the nine equations is given by the first row of Table 1 . Similarly, we can construct three additional sets of nine equations. Their solutions are also presented in Table 1. Clearly, there is no way in which these four solutions can be considered as compatible. Needless to say, the full set of 12 equations has no solution at all. This incompatibility is not accidental, it is generic. Using a different approach of analyzing the data, J.H. Bigelow came to a similar conclusion [50]. Apparently, including a model for the detector efficiency does not resolve the conflict between the experimental data of Weihs et al. and quantum theory of the EPRB thought experiment.

\section{DISCRETE EVENT SIMULATION}

The fact that the data produced by the EPRB experiment of Weihs et al. cannot be brought in harmony with the predictions of quantum theory for this experiment raises the question whether there exist (simulation) models of this experiment that produce the same kind of data sets as the real experiment and do not rely on concepts of quantum theory, yet reproduce the results of quantum theory of the EPRB experiment.

The possibility that such models exist was, to our knowledge, first pointed out by A. Fine [19]. The key of Fine's so-called synchronization model is the use of a filtering mechanism, essentially the time-coincidence window employed in laboratory EPRB experiments. Fine pointed out that such a filtering mechanism may lead to violations of the inequality $|S| \leq 2$, opening the route to a description in terms of locally causal, classical models. A concrete model of this kind was proposed by S. Pascazio who showed that his model approximately reproduces the correlation of the singlet state [56] with an accuracy that seems far beyond what is experimentally accessible to date. Later, Larson and Gill showed that Bell-like inequalities need to be modified in the case that the coincidences are determined by a time-window filter [57]. Finally, a time-tag model that exactly reproduces the results of quantum theory for the singlet and uncorrelated state was found [58-61].

A minimal, discrete-event simulation model of the EPRB experiment by Weihs et al. (see Fig. 1) requires a specification of the information carried by the particles, of the algorithm that simulates the source and the observation stations, and of the procedure to analyze the data.

- Source and particles: The source emits particles that carry a vector 
$\mathbf{S}_{n, i}=\left(\cos \left(\xi_{n}+(i-1) \pi / 2\right), \sin \left(\xi_{n}+(i-1) \pi / 2\right)\right)$, representing the polarization of the photons. This polarization is completely characterized by $\xi_{n}$ and the direction $i=1,2$ to which the particle moves. A uniform pseudo-random number generator is used to pick the angle $0 \leq \xi_{n}<2 \pi$. Clearly, the source emits two particles with a mutually orthogonal, hence correlated but otherwise random polarization.

- EOM: The EOM rotates the polarization of the incoming particle by an angle $\zeta$, that is $\alpha_{n, i} \equiv \operatorname{EOM}_{i}\left(\xi_{n}+(i-1) \pi / 2, \zeta_{i}\right)=\xi_{n}+(i-1) \pi / 2-\zeta_{i}$ symbolically. Mimicking the experiment of Weihs et al., we generate two binary uniform pseudorandom numbers $A_{i}=0,1$ and use them to choose the value of the angles $\zeta_{i}$, that is $\zeta_{1}=a\left(1-A_{1}\right)+A_{1}(a+\pi / 4)$ and $\zeta_{2}=b\left(1-A_{2}\right)+A_{2}(b+\pi / 4)$.

- Polarizing beam splitter: The simulation model for a polarizing beam splitter is defined by the rule

$$
x_{n, i}=\left\{\begin{array}{l}
1 \quad \text { if } \quad r_{n} \leq \cos ^{2} \alpha_{n, i} \\
0 \quad \text { if } \quad r_{n}>\cos ^{2} \alpha_{n, i}
\end{array}\right.
$$

where $0<r_{n}<1$ are uniform pseudo-random numbers. It is easy to see that for fixed polarization $\alpha_{n, i}=\alpha_{i}$, this rule generates events such that

$$
\lim _{N \rightarrow \infty} \frac{1}{N} \sum_{n=1}^{N} x_{n, i}=\cos ^{2} \alpha_{n, i}
$$

with probability one, showing that the distribution of events complies with Malus law.

- Time-tag model: The time-tag model of Pascazio is based on the assumption that the interaction between the photon and the detector follows an exponential probability law [56]. This model assumes that as the photon enters the detector at time $t=0$, it generates an electrical signal between $t$ and $t+d t$ with probability $p(t) d t=\lambda\left(\alpha_{n, i}\right) e^{-\lambda\left(\alpha_{n, i}\right) t} d t$ [56] or, in the notation used in the present paper, that the time tag is given by

$$
t_{n, i}=-\lambda\left(\alpha_{n, i}\right) \ln r
$$

where $\lambda\left(\alpha_{n, i}\right)$ is the inverse "life-time" of the photon-detector interaction which is assumed to depend on the polarization $\alpha_{n, i}$ [56], and $r$ is a uniformly distributed random number (which changes with each event).

As is well-known, as light passes through an EOM (which is essentially a tuneable wave plate), it experiences a retardation depending on its initial polarization and the rotation by the EOM. On the level of individual particles, in our time-tag model, we hypothesize that this delay is represented by the time tag

$$
t_{n, i}=\lambda\left(\alpha_{n, i}\right) r
$$

that is, the time tag is distributed uniformly over the interval $\left[0, \lambda\left(\alpha_{n, i}\right)\right]$ [58]. It has been shown that for $\lambda\left(\alpha_{n, i}\right)=T_{0} \sin ^{4} 2 \alpha_{n, i}$ this model, in combination with the 

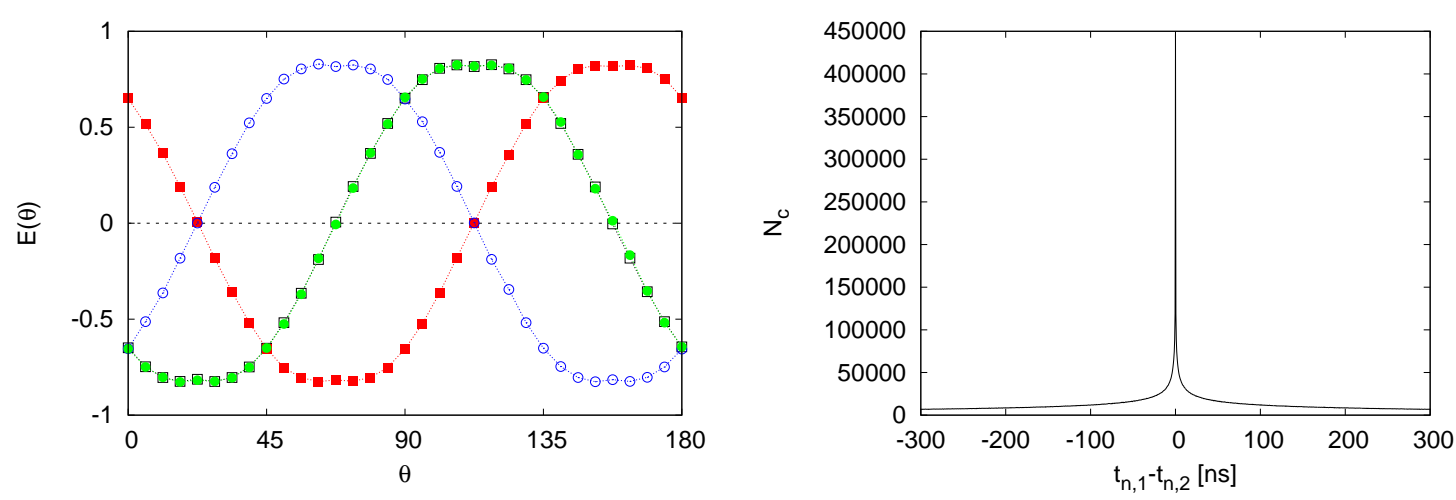

FIGURE 3. Simulation results produced by the locally causal, discrete-event simulation model using the time-tag model Eq. (11). Left: The two-particle correlation as a function of the angle $\theta$ for $W=$ 50ns. Open squares: $E(\theta)=E(\theta, \pi / 8)$; Open Circles: $E(\theta)=E(\theta+\pi / 4, \pi / 8)$; Solid squares: $E(\theta)=$ $E(\theta, 3 \pi / 8)$; Bullets: $E(\theta)=E(\theta+\pi / 4,3 \pi / 8)$; The dashed lines are guides to the eye only. Right: Histograms of the differences of the time-tags. The total number of pairs generated is $10^{6}$ and $T_{0}=2000 \mathrm{~ns}$.

model of the polarizing beam splitter, exactly reproduces the results of quantum theory of the EPRB experiments in the limit $W \rightarrow 0[59,60]$. We therefore adopt the expression $\lambda\left(\alpha_{n, i}\right)=T_{0} \sin ^{4} 2 \alpha_{n, i}$ for both time-tag models, leaving only $T_{0}$ as an adjustable parameter.

- Data analysis: The simulation algorithm generates the data sets $\Upsilon_{i}$, just as experiment does. We analyse these data sets in exactly the same manner as in the case of the experimental data.

This algorithm fully complies with Einstein's criterion of local causality on the ontological level: Once the particles leave the source, an action at observation station 1 (2) can, in no way, have a causal effect on the outcome of the measurement at observation station $2(1)$.

In Fig. 3 we present some typical simulation results for the two-particle correlation $E(a, b)$ and the distribution of time-tag differences, as obtained by using time-tag model Eq. (11). The single-particle averages $E_{1}(a)$ and $E_{2}(b)$ (data not shown) are zero up to the usual statistical fluctuations and do not show any statistically relevant dependence on $b$ or $a$, respectively, in concert with a rigorous probabilistic treatment of this simulation model [60].

For $W=2 \mathrm{~ns}$, the results for $E(a, b)$ fit very well to the prediction of quantum theory for the EPRB experiment. From these data (not shown), we extract $|S|=2.89$ and $|S|=2.82$ for Pascazio's and our time-tag model respectively. Note that for both models, the maximum value of $|S|$ is not limited by the quantum theoretical result and can, depending on the choice of $\lambda\left(\alpha_{n, i}\right)$, reach values that are very close to 4 [62]. For $W=50 \mathrm{~ns}$, the two models yield $|S|=2.63$ and $|S|=2.62$, respectively. Both results compare very well with the values extracted from the experimental data of Weihs et al., which for $W=50 \mathrm{~ns}$, are between 2 and 2.57. In all cases, the distribution of timetag differences is sharply peaked and displays long tails, in qualitative agreement with 
experiment [8].

\section{CONCLUSION}

It is highly unlikely that quantum theory describes the data of the EPRB experiment that we have analyzed. This suggests that in the real experiment, there may be processes at work which have not been identified yet. On the other hand, event-based simulation models provide a cause-and-effect description of real EPRB experiments at a level of detail which is not covered by quantum theory, such as the effect of the choice of the time-window. Some of these simulation models exactly reproduce the results of quantum theory of the EPRB experiment, indicating that there is no fundamental obstacle for an EPRB experiment to produce data that can be described by quantum theory. In any case, the popular statement that EPRB experiments agree with quantum theory does not seem to have a solid scientific basis yet.

\section{ACKNOWLEDGMENT}

We thank G. Weihs for providing us with the data sets of their EPRB experiments and S. Reuschel for help during the initial phase of the present work. We have profited from discussions with G. Adenier, J.H. Bigelow, K. De Raedt, K. Hess, A. Khrennikov, S. Reuschel, and G. Weihs. This work is partially supported by NCF, the Netherlands.

\section{REFERENCES}

1. C. A. Kocher, and E. D. Commins, Phys. Rev. Lett. 18, 575 - 577 (1967).

2. S. J. Freedman, and J. F. Clauser, Phys. Rev. Lett. 28, 938 - 941 (1972).

3. J. F. Clauser, and A. Shimony, Rep. Prog. Phys. 41, 1881 (1978).

4. A. Aspect, J. Dalibard, and G. Roger, Phys. Rev. Lett. 49, 1804 - 1807 (1982).

5. P. R. Tapster, J. G. Rarity, and P. C. M. Owens, Phys. Rev. Lett. 73, 1923 - 1926 (1994).

6. W. Tittel, J. Brendel, H. Zbinden, and N. Gisin, Phys. Rev. Lett. 81, 3563 - 3566 (1998).

7. G. Weihs, T. Jennewein, C. Simon, H. Weinfurther, and A. Zeilinger, Phys. Rev. Lett. 81, 5039 - 5043 (1998).

8. G. Weihs, Ein Experiment zum Test der Bellschen Ungleichung unter Einsteinscher Lokalität, Ph.D. thesis, University of Vienna (2000), http://www.quantum.univie.ac.at/ publications/thesis/gwdiss.pdf.

9. G. Weihs, "A Test of Bell's Inequality with Spacelike Separation," in Foundations of Probability and Physics-4, edited by G. Adenier, C. A. Fuchs, and A. Y. Khrennikov, AIP Conference Proceedings, Melville, New York, 2007, vol. 889, pp. 250 - 260.

10. M. A. Rowe, D. Kielpinski, V. Meyer, C. A. Sackett, W. M. Itano, C. Monroe, and D. J. Wineland, Nature 401, 791 - 794 (2001).

11. D. Fatal, E. Diamanti, K. Inoue, and Y. Yamamoto, Phys. Rev. Lett. 92, 037904 (2004).

12. H. Sakai, T. Saito, T. Ikeda, K. Itoh, T. Kawabata, H. Kuboki, Y. Maeda, N. Matsui, C. Rangacharyulu, M. Sasano, Y. Satou, K. Sekiguchi, K. Suda, A. Tamii, T. Uesaka, and K. Yako, Phys. Rev. Lett. 97, 150405 (2006).

13. A. A. Hnilo, M. D. Kovalsky, and G. Santiago, Found. Phys. 37, 80 - 102 (2007).

14. J. S. Bell, Speakable and unspeakable in quantum mechanics, Cambridge University Press, Cambridge, 1993.

15. L. E. Ballentine, Quantum Mechanics: A Modern Development, World Scientific, Singapore, 2003. 
16. J. Bell, Physics 1, 195 - 200 (1964).

17. L. de la Peña, A. Cetto, and T. Brody, Lett. Nuovo Cim. 5, 177 - 181 (1972).

18. A. Fine, Synthese 29, $257-289$ (1974).

19. A. Fine, Synthese 50, $279-294$ (1982).

20. A. Fine, Phys. Rev. Lett. 48, $291-295$ (1982).

21. A. Fine, J. Math. Phys. 23, $1306-1310$ (1982).

22. W. M. de Muynck, Phys. Lett. A 114, $65-67$ (1986).

23. M. Kupczyński, Phys. Lett. A 116, 417 - 419 (1986).

24. E. T. Jaynes, "Clearing up mysteries - The original goal," in Maximum Entropy and Bayesian Methods, edited by J. Skilling, Kluwer Academic Publishers, Dordrecht, 1989, vol. 36, p. 1.

25. T. Brody, The Philosphy Behind Physics, Springer, Berlin, 1993.

26. I. Pitowsky, Brit. J. Phil. Sci. 45, 95 - 125 (1994).

27. A. Fine, The Shaky Game: Einstein Realism and the Quantum Theory, University of Chicago Press, Chicago, 1996.

28. A. Y. Khrennikov, Interpretations of Probability, VSP Int. Sc. Publishers, Utrecht, 1999.

29. L. Sica, Opt. Comm. 170, 55 - 60 (1999).

30. W. De Baere, A. Mann, and M. Revzen, Found. Phys. 29, 67 - 77 (1999).

31. K. Hess, and W. Philipp, Proc. Natl. Acad. Sci. USA 98, 14228 - 14233 (2001).

32. K. Hess, and W. Philipp, "Bell's theorem: Critique of Proofs with and without Inequalities," in Foundations of Probability and Physics-3, edited by A. Khrennikov, AIP Conference Proceedings, Melville, New York, 2005, vol. 750, p. 150.

33. L. Accardi, "Some loopholes to save quantum nonlocality," in Foundations of Probability and Physics-3, edited by A. Khrennikov, AIP Conference Proceedings, Melville, New York, 2005, vol. 750, p. 21.

34. A. F. Kracklauer, "Bell's Inequalities and EPR-B Experiments: Are They Disjoint?," in Foundations of Probability and Physics-3, edited by A. Khrennikov, AIP Conference Proceedings, Melville, New York, 2005, vol. 750, p. 219.

35. E. Santos, Phil. Mod. Phys. 36, $544-565$ (2005).

36. E. Loubenets, Found. Phys. 35, $2051-2072$ (2005).

37. M. Kupczyński, J. Russ. Las. Res. 26, $514-523$ (2005).

38. P. Morgan, J. Phys. A 39, 7441 - 7445 (2006).

39. A. Y. Khrennikov, "A Mathematicians Viewpoint to Bell's Theorem," in Foundations of Probability and Physics-4, edited by G. Adenier, C. A. Fuchs, and A. Y. Khrennikov, AIP Conference Proceedings, Melville, New York, 2007, vol. 889, p. 7.

40. G. Adenier, and A. Y. Khrennikov, J. Phys. B: At. Mol. Opt. Phys. 40, $131-141$ (2007).

41. T. M. Nieuwenhuizen, "Where Bell Went Wrong," in Foundations of Probability and Physics - 5, edited by L. Accardi, G. Adenier, C. Fuchs, G. Jaeger, A. Khrennikov, J. A. Larsson, and S. Stenholm, AIP Conference Proceedings, Melville and New York, 2009, vol. 1101, p. 127.

42. A. Matzkin, "Is Bell's theorem relevant to quantum mechanics? On locality and non-commuting observables," in Foundations of Probability and Physics - 5, edited by L. Accardi, G. Adenier, C. Fuchs, G. Jaeger, A. Khrennikov, J. A. Larsson, and S. Stenholm, AIP Conference Proceedings, Melville and New York, 2009, vol. 1101, p. 339.

43. K. Hess, K. Michielsen, and H. De Raedt, Europhys. Lett. 87, 60007 (2009).

44. A. Y. Khrennikov, Contextual Approach to Quantum Formalism, Springer, Berlin, 2009.

45. D. A. Graft, Phys. Essays 22, 534-542 (2009).

46. A. Khrennikov, J. Comp. Theor. Nanosci. 8, 1006-1010 (2011).

47. T. Nieuwenhuizen, Found. Phys. 41, 580-591 (2011).

48. H. De Raedt, K. Hess, and K. Michielsen, J. Comp. Theor. Nanosci. 8, 1011 - 1039 (2011).

49. A. Hnilo, A. Peuriot, and G. Santiago, Found. Phys. Lett. 15, 359 - 371 (2002).

50. J. Bigelow (2009), URL http: / / arxiv. org/abs / $0906.5093 \mathrm{v} 1$.

51. M. Agëro, A. Hnilo, M. Kovalsksy, and M. Larotonda, Eur. Phys. J. D 55, 705 -709 (2009).

52. D. Bohm, Quantum Theory, Prentice-Hall, New York, 1951.

53. A. Einstein, A. Podolsky, and N. Rosen, Phys. Rev. 47, 777 - 780 (1935).

54. J. F. Clauser, and M. A. Horne, Phys. Rev. D 10, 526 - 535 (1974).

55. W. H. Press, B. P. Flannery, S. A. Teukolsky, and W. T. Vetterling, Numerical Recipes, Cambridge University Press, Cambridge, 2003. 
56. S. Pascazio, Phys. Lett. A 118, 47 - 53 (1986).

57. J. A. Larsson, and R. D. Gill, Europhys. Lett. 67, 707 - 713 (2004).

58. K. De Raedt, K. Keimpema, H. De Raedt, K. Michielsen, and S. Miyashita, Euro. Phys. J. B 53, 139 - 142 (2006).

59. K. De Raedt, H. De Raedt, and K. Michielsen, Comp. Phys. Comm. 176, 642 - 651 (2007).

60. S. Zhao, H. De Raedt, and K. Michielsen, Found. of Phys. 38, 322 - 347 (2008).

61. K. Michielsen, F. Jin, and H. De Raedt, J. Comp. Theor. Nanosci. 8, 1052 - 1080 (2011).

62. H. De Raedt, K. De Raedt, K. Michielsen, K. Keimpema, and S. Miyashita, J. Comp. Theor. Nanosci. 4, 957 - 991 (2007). 